\title{
Korrespondenzen.
}

\section{Die Kampfesweise des Hauptverbandes deutscher Kranken-} kassen.

In Nr.32 der "Ortskrankenkasse“, Organ des Hauptverbandes deutşcher Krankenkassen E. "V. Dresden, vom 7. V1ll., wird in einem Artikel: „Der Kampf gegen die Selbstabgabe der Krankenkassen" gegen die chemischpharmazeutische Großindustrie folgender verleumderischer Anwurf gerichtet:

"Geld genug ist für eine solche Propaganda vorhanden. Erfuhren wir doch kürzlich von prominenter Seite, daB den Apothekern 10000 Goldmark von der chemisch-pharmazeutischen GroBindustrie zur Verfügung gestellt wurden, um die Heilmittelversorgung deutscher Krankenkassen A.-G. lahmzulegen."

Unserer Forderung, eine Erklärung abzugeben:

„daB aus dem Kreise unseres Verbandes weder den Apothekern im allgemeinen noch irgendeiner Apothekerorganisation Geld zur Verfügung gestellt worden ist, ganz hesonders nicht zur Lahmlegung der Heilmittelversorgung deutscher Krankenkassen“, wich die Schriftleitung zunächst mit der Bemerkung aus: „An1 der wortgetreuen Darstellung unseres Gewährsmannes zu zweifeln, haben wir vorläufig noch keinen AnlaB“, obwohl damals schon die ApothekerZeitung Nr.66 vom 16. V111. vorlag, in der der Deutsche Apotheker-Verein die Versicherung abgab, ,dab die Behauptung, die chemisch-pharmazeutische Großindustrie habe 10000 Goldmark zur Verfügung gestellt, soweit sie den Deutschen Apotheker-Verein und seinen Vorstand angeht, frei erfunden ist“".

Erst auf die Androhung weiter zu unternehmender Schritte mit Fristsetzung bis 5. 1X. fühlte sich die "Ortskrankenkasse“ bewogen, unter dem Stichwort „Allerlei Berichtigungen“ unsere Erklärung abzudrucken, jedoch unter Hinzufügung folgender Bemerkungen:

„Wir wollen uns nicht mit langatmigen Polemiken aufhalten, sondern hierher setzen, was uns zu diesen Berichtigungen unser Gewährsmann schreibt, für dessen Zuverlässigkeit wir bürgen können:

,lch hatte an den hiesigen Großhändler für chemische Artikel, Herrn ..., die Anfrage gerichtet, ob er mir 2000 Pyramidon-Tabletten liefern könne. Derselbe sagte die Lieferung zu und wollte dieselben telegraphisch bestellen. Als die Tabletten nach einigen Tagen noch nicht eingetroffen waren, fragte ich an einem Samstag Mittag gegen 2 Uhr in seiner Wohnung telephonisch an, wie es mit der Lieferung stände. Herr .... antwortete in einem ganz aufgeregten Tone, daB er die Tabletten nicht liefern werde, daB er auch telegraphische Anweisung an den Fabrikanten, Herrn ... nach ... gegeben habe, $\mathrm{daB}$ wir von dort die Tabletten nicht erhalten würden. Im übrigen würde er die Heilmittelvertriebsgesellschaft bekämpfen, wo immer er könne. $\mathrm{Er}$ habe der Apothekervereinigung 10000 Goldmark zur Verfügung gestelit zur Bekämpfung der Heilmittelvertriebsgesellschaft, und wir würden es bald zu spüren bekommen.

Die Namen der betreffenden Firmen haben wir aus diesem Schreiben ausgelassen, können sie aber jederzeit nennen. Der Leser mag sich nun selbst ein Urteil bilden.“

Da man ohne weiteres annehmen darf, daß der Vorstand des Hauptverbandes deutscher Krankenkassen und seine Schriftleiter den Unterschied zwischen Händlern und Fabrikanten kennen, auch daB man Händler gemeinhin nicht zur Großindustrie zu rechnen pflegt, daß ihnen zudem die Mitglieder unseres Verbandes wohl bekannt sind, kann in dem Zusatz zu unserer Richtigstellung nur eine lrreführung der Oeffentlichkeit angenommen werden.

Nach den mit der Schriftleitung der "Ortskrankenkasse" gemachten Erfahrungen kann es uns nicht verdacht werden, wenn wir auf weitere Berichtigungen in diesem Blatt verzichten.

Verband der chem.-pharm. GroB-Industrie E. V. 